\title{
Integrated Production Planning and Quality Control for Linear Production Systems Under Uncertainties of Cycle Time and Finished Product Quality
}

\author{
Oussama Ben-Ammar ${ }^{\mathrm{a}}$, Belgacem Bettayeb ${ }^{\mathrm{b} *}$ and Alexandre Dolgui ${ }^{\mathrm{a}}$ \\ ${ }^{a}$ LS2N, IMT Atlantique, 4 rue Alfred Kastler, 44300 Nantes, France; \\ ${ }^{b}$ LINEACT Lab., CESI Group, 1 Rue G. Marconi, 76130 Mont-Saint-Aignan, France.
}

(Revised version submission)

\begin{abstract}
This work considers serial production systems with several process steps and a possible quality control at final step. It deals with the problem of optimizing planned lead time when the real lead time for each process is stochastic and the finished product quality is uncertain unless it is inspected. Three analytical models are proposed aiming to minimize the expected total cost, which is composed of the inventory and backlogging costs for the finished product and quality costs associated with inspection and non-conformities. These models correspond to three quality control policies: i) without quality control, ii) with quality control but without taking into account the inspection duration when optimizing the planned lead time and iii) with quality control and with considering the inspection duration when optimizing the planned lead time. Based on the results, it can be highlighted the economic advantage of integrating quality control at the early stage of supply and production planning decisions for some cost parameters conditions. The robustness of the proposed models is also analyzed regarding the variance of the probability distributions of the lead times.
\end{abstract}

Keywords: Production planning; Cycle time uncertainty; Imperfect linear production process; Quality control.

\section{Introduction}

Uncertainty is one of the most challenging aspects in supply chain (SC) design and planning, and operations management. It is attracting more and more researches because of the growing need of efficient, sustainable, agile and resilient systems and organisations at different stages of the whole supply chain of products and services. In fact, the challenge is how to deal with uncertainty in order to mitigate its impacts on the performances of the supply chain. The difficulties caused to

*Corresponding author. Email: bbettayeb@cesi.fr 
companies lie in the decision making concerning several activities such as production planning, quality control, inventory regulation, and demand satisfaction (Tang 1990).

Several research works have focused on identifying the sources and how to manage uncertainty and risks in SC. In the literature review of Simangunsong, Hendry, and Stevenson (2012), 14 sources of uncertainty have been reported and divided into three groups: i) internal organisation uncertainty; ii) internal supply-chain uncertainty and iii) external uncertainties. Managing uncertainty in SC consists of defining approaches and techniques to model and to decide appropriate actions aiming to reduce and/or cope with uncertainty. Moreover, new contexts and sources of uncertainty encountered may constrain companies to review their SC redesign strategies to be adopted (Van der Vorst and Beulens 2002).

In pull- and hybrid pull-push-based SC systems, such as Engineer-To-Order (ETO), Make-ToOrder (MTO) and Assemble-To-Order (ATO), associated with Just-In-Time (JIT) manufacturing, the production process of a product begins only after a confirmed customer order is received. This enables firms to launch production based on customer orders with a specific quantity and due date, and, consequently, inventory of finished products can be reduced to zero. Nevertheless, the effectiveness of this kind of environment can be considerably affected by the variation of some inherently-uncertain parameters, often considered as deterministic, whose effects may propagate and intensify along the subsequent echelons of the SC. For instance, the production process can be interrupted by a machine breakdown, some components replenishment lead times can be significantly longer than planned ones, or a quality issue can arise which lead to additional time to inspect/rework/reproduce impacted semi-finished or finished products. Therefore, the occurrence of this kind of events may delay the delivery of finished products and increase production costs.

In this paper, we consider a serial production system in ETO environment where a tailored finished product fulfil a specific customer request and pass through successive processing steps. We precise that all process steps (work-stations, assembly units...) are considered independent enterprises (or partners) where we have no control on internal decisions to coordinate activities inside them. All what we know is an estimation of the lead time of each partner. Consequently, we are responsible for providing the costumer with good quality products and respecting the delivery date. During the contract negotiation step, (1) a client orders a quantity of a specific product. Then, (2) we design the corresponding successive processing steps, (3) we estimate the total lead time, (4) we negotiate (i) the planned due date for client delivery, (ii) the unit non-conformity cost and (iii) the unit backlogging cost. According to the negotiated parameters and using our approach, we can decide the date when the first process starts and which quality control policy to adopt. We precise that, at our decision level, only the distribution of probability of each partner's lead time 
is known. It is obtained with statistics or estimated by partner himself, and includes all aspects such as assembly durations, transportation, waiting times, etc. This assumption is often used in literature ((May, Atkinson, and Ferrer 2017), (Kuang, Hu, and Ko 2016), (Ding, Benyoucef, and Xie 2005), (Petrovic, Roy, and Petrovic 1998)).

This paper is a revised and extended version of (Ben-Ammar, Bettayeb, and Dolgui 2017) that aims to optimize planned lead times while minimising the expected total cost of a linear production process, where the lead times between process steps are stochastic and the finished product quality is uncertain. Three mathematical models were developed and tested to evaluate different quality control policies. In this extended version, the mathematical models are refined and the experiments are extended by including additional analysis of the effects of several parameters and by providing an analysis of robustness regarding the variation of lead times' variance.

The rest of the paper is organized as follows. In section 2 , and without being exhaustive on this topic, we will focus on some relevant works related to linear supply and production planning integrating quality control. The problem description and the general assumptions are given in section 3. The forth section details the analytical models corresponding to three different quality control policies and explains the associated optimization approach. Section 5 presents the experimental results, compares the economical performances of each quality policy and analysis the effects of some parameters. The paper ends by giving the conclusion and the perspectives of this work.

\section{Related works}

One of the first study of lead time uncertainty is presented in Whybark and Williams (1976). A simulation model is developed to suggest that safety lead times may perform better than safety stocks in a multi-level serial production system when the production and replenishment times are random. Earlier, a one-stage and one-period planning model under stochastic processing time was proposed by Weeks (1981). Author considered tardiness and holding costs and proved that this problem is equivalent to the standard 'Newsboy' problem. A few years later, Yano (1987a) proposed an analytical approach to model a two-stage systems and to determine the planned lead times while minimizing holding and tardiness costs. She assumed a Lot-For-Lot policy, a stochastic procurement and processing times, and a deterministic demand. This model has been extended in Yano (1987b) to model three-stage systems and to integrate a replenishment cost. The objective function was to minimize the expected total cost, which is composed of a holding and tardiness costs at each stage, and a tardiness cost for the finished product. The author underlined that, for more than three process steps, modelling the problem seemed to be difficult. It is only after many year 
that Elhafsi (2002) overcame this barrier by proposing an analytical model based on recursivity. Author proved the convexity of the objective function and proposed a heuristic based on dynamic programming to find approximate solutions of good quality in an acceptable computation time.

In inventory control process, Kim et al. (2004) consider a one product, a one-period planning and an Erlang-distributed lead times to optimize the expected total cost, which is composed of ordering, holding and tardiness costs. Based on the analytical formulation, approximate solutions are proposed and compared to the optimal ones for the case where the prior information on the lead time distribution is available, and another case where no information exists. For uncertain lead times, authors proved the effectiveness and the robustness of the proposed method and specified how costs could be reduced. Two years later, the same problem and the bull-whip effect was studied by Kim et al. (2006) when both demand and lead times are stochastic. By using an analytical method to model a multi-stage supply chain, author proved that prior information on lead times variability could be very helpful to control the bull-whip effect.

As reported by (Jansen et al. 2018; Dolgui et al. 2013), the literature contains few publications considering serial supply and production planning under uncertain lead times (see Table 1). The most of existing work investigates the uncertainty of lead time for one item or for assembly systems. Readers can refer to (Dolgui et al. 2013; Dolgui and Prodhon 2007; Guide and Srivastava 2000) where more complete literature reviews are provided concerning supply planning dealing with uncertainty on lead times. For other sources of uncertainty (demand, capacity, cost, etc.) readers can refer to the surveys of Aloulou, Dolgui, and Kovalyov (2014) and Díaz-Madroñero, Mula, and Peidro (2014).

On another hand, in the last few decades, there has been a growing interest in integrating quality control in supply and production planning. In fact, the quality should be rigorously checked before delivering the finished product. In this way, the company ensures customer satisfaction and avoid excessive product returns. In this regard, Bettayeb, Bassetto, and Sahnoun (2014) proposed an exposure-based quality control planning approach to prevent excessive scraps in semiconductor manufacturing under limited quality control capacity constraint. Moreover, it is also necessary to find a good compromise between the inspection costs and the cost of non-conformities. In this regard, Colledani and T. (2006) investigate the interaction between quality control system and production system and show how they impact each others performances. They underline the necessity of jointly considering quality and logistics requirements while designing a production system. The work of Rosenblatt and Lee (1986) studies the relationship and the economic impact of the interaction between production planning, quality of products and the deterioration of the production process. The considered production process has two possible states in which it generates 
Table 1.: Linear production systems under uncertainty of lead times

\begin{tabular}{|c|c|c|c|c|c|c|}
\hline \multirow[b]{2}{*}{ 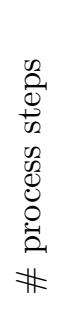 } & \multirow[b]{2}{*}{ References } & \multirow[b]{2}{*}{ Criteria } & \multicolumn{3}{|c|}{ Type of system } & \multirow[b]{2}{*}{ Comments } \\
\hline & & & 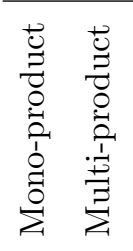 & 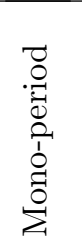 & 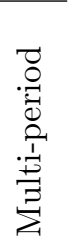 & \\
\hline \multirow{5}{*}{1} & Weeks (1981) & $C_{H} ; C_{T}$ & $\mathrm{x}$ & $\mathrm{x}$ & & $\begin{array}{l}\text { Newsboy Formulae; Con- } \\
\text { tinuous lead times. }\end{array}$ \\
\hline & $\begin{array}{l}\text { Matsuura and Tsubone (1993); Mat- } \\
\text { suura, Tsubone, and Kanezashi (1996) }\end{array}$ & CRV & $\mathrm{x}$ & $\mathrm{x}$ & & $\begin{array}{l}\text { MTO environment; Queu- } \\
\text { ing models. }\end{array}$ \\
\hline & Buzacott and Shanthikumar (1994) & $C_{H} ; C_{T}$ & $\mathrm{x}$ & & $\mathrm{x}$ & $\begin{array}{l}\text { Stochastic modelling; } \\
\text { Safety stock versus safety } \\
\text { time }\end{array}$ \\
\hline & Kim et al. (2004) & $C_{H} ; C_{T}$ & $\mathrm{x}$ & & $\mathrm{x}$ & $\begin{array}{l}\text { Erlang-distributed lead } \\
\text { times; (s,Q) inventory } \\
\text { model; } \quad \text { approximate } \\
\text { solutions }\end{array}$ \\
\hline & Kim et al. (2006) & BWE & $\mathrm{x}$ & & $\mathrm{x}$ & $\begin{array}{l}\text { Normal-distributed lead } \\
\text { time; stochastic demand; } \\
\text { (R,S) inventory model }\end{array}$ \\
\hline $2-3$ & Yano (1987a,b) & $C_{H} ; C_{T}$ & $\mathrm{x}$ & $\mathrm{x}$ & & $\begin{array}{l}\text { Analytical approach, con- } \\
\text { tinuous lead times }\end{array}$ \\
\hline \multirow[t]{3}{*}{$\mathrm{m}$} & Gong, de Kok, and Ding (1994) & $C_{H} ; C_{T}$ & $\mathrm{x}$ & $\mathrm{x}$ & & $\begin{array}{l}\text { Holding cost for each pro- } \\
\text { cess; tardiness cost for the } \\
\text { finished product }\end{array}$ \\
\hline & Elhafsi (2002) & $C_{H} ; C_{T} ; C_{E}$ & $\mathrm{x}$ & $\mathrm{x}$ & & $\begin{array}{l}\text { Planned start time for } \\
\text { each process; recursive re- } \\
\text { lations }\end{array}$ \\
\hline & This paper & $C_{H} ; C_{T} ; C_{Q}$ & $\mathrm{x}$ & $\mathrm{x}$ & & $\begin{array}{l}\text { Newsboy Formula; dis- } \\
\text { crete lead times; quality } \\
\text { control }\end{array}$ \\
\hline
\end{tabular}

$C_{T}$ : Tardiness cost, $C_{H}$ : Holding cost, $C_{E}$ : Earliness cost, $C_{Q}$ : Quality cost, $C R V$ : Capacity Requirements variations, $B W E$ : Bull-whip effect

different rates of defectives. The objective is to determine the economic production quantities of this kind of production process while minimizing the total annual cost. The optimal production run time is determined for two deterioration models and multi-state deterioration. Recently, Bettayeb, Brahimi, and Lemoine (2017) proposed an integrated model for single item lot sizing and quality control planning. The objective is to minimize the total cost while ensuring a given level of outgoing quality. The total cost is composed of holding, setup, production and inspection costs.

We note also that there are other interesting works on the trade-off between planned lead times and quality issues (number of rejects) in (Dolgui, Levin, and Louly 2005) and (Schemeleva, Delorme, and Dolgui 2018), but the decision variables in that works were the lot sizes and their sequencing order. 
As it is shown in Table 1, no publication has been found in the literature that takes into account both uncertainties of lead times and finished product quality in linear production systems. For such a system, the research question is about how to efficiently coordinate production planning with quality control decisions. In this work, we seek to fulfill this research gap by proposing an integrated model of supply planning optimization and quality control of imperfect linear production systems with stochastic lead times.

\section{Problem description and formulation}

The problem under consideration in this paper concerns the optimization of production planning of an imperfect linear production process where the lead times of the production steps are stochastic and quality control can be used to decrease non-quality costs. The objective is to evaluate the effect of quality issues on the total cost of such a system and to analyse the opportunity of integrating quality control activities in the optimal release date calculation. Before detailing the problem and general assumptions, the following are the notations that will be used in next sections. All costs are expressed in monetary unit.

\section{Parameters}

$C_{P} \quad$ Production cost

$c_{i} \quad$ Unit inspection cost

$c_{n c} \quad$ Unit non-conformity cost

$C_{Q} \quad$ Quality cost: the sum of inspection and non-conformity costs

$S R \quad$ Sampling rate, i.e. the proportion of products inspected or the probability that a given product is being inspected

$c_{h} \quad$ Unit inventory holding cost per time unit

$c_{t} \quad$ Unit tardiness penalty per time unit

$T \quad$ Due date

$q_{0} \quad$ Proportion of non-conforming finished products generated by the whole production process. 
$m \quad$ Number of production process operations

$L_{j} \quad$ Lead time of operation $j \in\{1,2, \ldots, m\}$, it is a discrete random variable which varies between $l_{j}$ and $u_{j}$

$L \quad$ Total lead time, i.e. $L=\sum_{j=1}^{m} L_{j}$

$U \quad$ Longest possible duration of total lead time, i.e. $U=\sum_{j=1}^{m} u_{j}$

$V \quad$ Shortest possible duration of total lead time, i.e. $V=\sum_{j=1}^{m} l_{j}$

$t_{i} \quad$ Unit inspection duration

\section{Decision variables}

$X \quad$ Order release date

\section{Functions}

$\operatorname{Pr} \llbracket A \rrbracket$ Probability of $A$

$E \llbracket A \rrbracket \quad$ Expected value of $A$

$F_{i}($.$) \quad Convolution cumulative distribution function of \sum_{j=i}^{m} L_{j} \forall i \in\{1, \ldots, m\}$

$z^{+} \quad \max (z, T)$

$z^{-} \quad \min (z, T)$

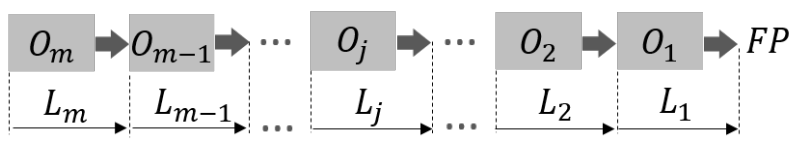

Figure 1.: Linear production process

The considered production system is composed of $m$ process operations (Fig.1), which are consecutively executed to obtain the finished product $F P$. Without loss of generality, we assume that:

- The demand is known and equal to one;

- The lead time $L_{j}$ of a process step $j$ is an independent discrete random variable with known probability distribution and finite upper value. It includes capacity constraints, machine breakdowns, stochastic variations on operation processing times, etc.; 
- No decision is possible on the start date for intermediate process steps;

- The average outgoing quality level $q_{0}$, before the final inspection of the finished product, is known. It is the result of the succession of different and independent quality control policies applied within each process step;

- A final error-free inspection of the finished product (e.g. functional testing) may be done after the last process step. It is used to control/adjust the quality level delivered to the final customer;

- The rework of non-conforming finished product is instantaneous and cost-free, i.e. rework duration and cost are neglected compared to those of inspection.

The product starts to be processed at time $X$, to be decided, and the fished product is available at $T_{F P}=X+L$, after accumulating the $m$ random lead times $\left(L=L_{1}+L_{2}+\cdots+L_{m}\right)$. Note that $T_{F P}$ is a random variable because of the randomness of $L$. Apart from the processing cost (omitted here), and depending on the due date and the effective delivery date of the finished product, the generated production cost is either proportional to the holding cost, if the product is finished before the due date, or proportional to the tardiness penalty, if it is available after the due date, or equal to zero, if it is finished just in time.

\section{Analytical models and optimization approach}

This section contains three models, each of them corresponds to a different policy regarding the quality control (see Figure 2). In the first policy, no quality control is performed by the producer who assumes a penalty for each non-conforming product delivered to the customer. In the second policy, the quality control is performed at the end of production process but only the lead times are taken into account in the optimization of the order release date. In the last policy, the quality control and the lead time are both taken into account when optimizing the order release date.

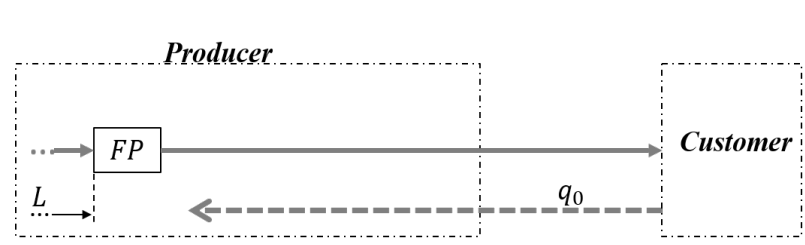

(a) Policy $\pi_{0}$ : without quality control

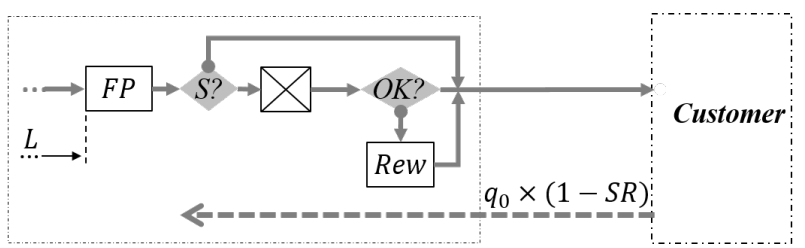

(b) Policies $\pi_{1} \& \pi_{2}$ : with quality control performed by the producer

Figure 2.: Quality control policies 


\subsection{Policy $\left(\pi_{0}\right):$ No quality control}

This policy corresponds to the case where no quality control is performed during the production process. However, for each non-conforming product delivered to the customer, the producer pays a non-conformity penalty. The total cost is composed of the costs of production $\left(C_{P}^{0}\right)$ and quality $\left(C_{Q}^{0}\right)$ :

$$
T C^{0}(X, L)=C_{P}^{0}+C_{Q}^{0}
$$

where $C_{P}^{0}$ is equal to the sum of the inventory holding cost $\left(C_{H}\right)$ and the tardiness cost $\left(C_{T}\right)$, which are given below:

- if the finished product is available before $T$, it will be stored. The corresponding inventory holding $\operatorname{cost} C_{H}$ is equal to:

$$
C_{H}=c_{h} \times\left(T-T_{F P}^{-}\right)
$$

- if the finished products are delivered after the due date $T$, there will be a stockout. Then, tardiness cost is equal to:

$$
C_{T}=c_{t} \times\left(T_{F P}^{+}-T\right)
$$

The cost of quality, in this case, corresponds to non-conformity penalty, which is equal to:

$$
C_{Q}^{0}=q_{0} \times c_{n c}
$$

The total cost $T C^{0}(X, L)$ is a discrete random variable because of the randomness of the total lead time $L$. The latter being the sum of the random variables $L_{j}$, each of which varies between $l_{j}$ and $u_{j}$, the total cost has a finite number of possible values.

Property 1. The expression of the expected value of the total cost of policy $\pi_{0}$ is:

$$
E \llbracket T C^{0}(X, L) \rrbracket=c_{h} \times \sum_{s=V}^{T-1-X} F_{1}(s)+c_{t} \times\left(X+U-T-\sum_{s=T-X}^{U-1} F_{1}(s)\right)+q_{0} \times c_{n c}
$$

where $T-U \leq X \leq T-V$ and $F_{i}$ is the convolution cumulative distribution function of $L_{i}+$ 
$L_{i+1}+\cdots+L_{m}$, which is defined as below:

$$
F_{i}(s)= \begin{cases}\operatorname{Pr} \llbracket L_{m} \leq s \rrbracket & \text { if } i=m \\ \sum_{\substack{v_{i}+w_{i}=s \\ v_{i}+w_{i} \in \mathbb{N}}} \operatorname{Pr} \llbracket L_{i}=v_{i} \rrbracket \times F_{i+1}\left(w_{i}\right) & \forall i=1, \ldots, m-1\end{cases}
$$

Proof. From expressions (1), (2) and (3), we have:

$$
E \llbracket T C^{0}(X, L) \rrbracket=E \llbracket C_{P}^{0}(X, L) \rrbracket+q_{0} \times c_{n c}=c_{h} \times\left(T-E \llbracket T_{F P}^{-} \rrbracket\right)+c_{t} \times\left(E \llbracket T_{F P}^{+} \rrbracket-T\right)+q_{0} \times c_{n c}
$$

Let $\Gamma$ be a positive and finite discrete random variable and $C D F_{\Gamma}$ its cumulative distribution function. The expression of $\Gamma$ 's expected value can be written in the following form:

$$
E \llbracket \Gamma \rrbracket=\sum_{s \geq 0}(1-\operatorname{Pr} \llbracket \Gamma \leq s \rrbracket)=\sum_{s \geq 0}\left(1-C D F_{\Gamma}(s)\right)
$$

Thus, knowing that $X+L$ does not depend on $T$, we have:

$$
E \llbracket T_{F P}^{+} \rrbracket=\sum_{s \geq 0}\left(1-\operatorname{Pr} \llbracket T \leq s \rrbracket \times \operatorname{Pr} \llbracket T_{F P} \leq s \rrbracket\right)
$$

Moreover, knowing that $\operatorname{Pr} \llbracket T \leq s \rrbracket=0 \forall s \in[0, T[$ and $\operatorname{Pr} \llbracket T \leq s \rrbracket=1 \forall s \geq T$, then:

$$
E \llbracket T_{F P}^{+} \rrbracket=T+\sum_{s \geq T}\left(1-\operatorname{Pr} \llbracket T_{F P} \leq s \rrbracket\right)=T+\sum_{s \geq T}\left(1-F_{1}(s-X)\right)
$$

The finished product is available at period $U+X$ at latest. Thus, $T_{F P}^{+}$varies between $T$ and $X+U$. Then:

$$
E \llbracket T_{F P}^{+} \rrbracket=U+X-\sum_{s=T-X}^{U-1} F_{1}(s)
$$

In the same way, using the fact that the finished product can never be available before period $X+V$, i.e. $T_{F P}^{-} \in[X+V, T]$, it can be easily proved that:

$$
E \llbracket T_{F P}^{-} \rrbracket=T-\sum_{s=V}^{T-1-X} F_{1}(s)
$$


Then, equation (5) can be deduced.

The expected total cost expressed in (5) constitutes a non-linear objective function to be minimized. An exact method based on the well-known Newsboy formula is used to solve this problem in polynomial time. This method is detailed in the next proposition and an illustrative example is given in section 5.1

Proposition 1. The method based on Newsboy formula gives the optimal order release date of the reference case $X_{0}^{*}$ which should satisfy the following optimality condition:

$$
F_{1}\left(T-X_{0}^{*}-1\right) \leq \frac{c_{t}}{c_{h}+c_{t}} \leq F_{1}\left(T-X_{0}^{*}\right)
$$

were $F_{1}$ is the convolution cumulative distribution function of the total lead time and $c_{t}$ and $c_{h}$ are the unit time tardiness cost and the unit time holding cost, respectively.

Proof. See (Ben-Ammar et al. 2014) or (Ben-Ammar, A., and Wu 2018).

\subsection{Policy $1\left(\pi_{1}\right)$ : Separated afterwards quality control planning}

With this policy, we suppose that one quality inspection is performed just after the last production operation. A proportion of finished products is randomly sampled and inspected. The non-conforming products detected by inspection are repaired with no-extra cost. Thus, the total cost is composed of production and quality costs, denoted by $C_{P}^{1}$ and $C_{Q}^{1}$, respectively.

$$
T C^{1}(X, L, S R)=C_{P}^{1}+C_{Q}^{1}
$$

Note that, in this case, the actual production $\operatorname{cost} C_{P}^{1}$ is equal to $C_{P}^{0 *}-\Delta_{h}+\Delta_{t}$, where:

- $C_{P}^{0 *}$ is the optimal planned production cost associated to optimal order release date $X_{0}^{*}$;

- $\Delta_{h}$ is the holding cost reduction corresponding to the time the product is being inspected:

$$
\Delta_{h}=c_{h} \times \max \left(\min \left(I ; T-T_{F P}\right) ; 0\right)
$$

where $I=t_{i} \times S R$ is the inspection duration which corresponds to the quality control strategy in place; 
- $\Delta_{t}$ is the tardiness cost increase due to the product inspection:

$$
\Delta_{t}=c_{t} \times\left(I-\max \left(\min \left(I ; T-T_{F P}\right) ; 0\right)\right)
$$

The quality cost $C_{Q}^{1}$ is composed of the cost of inspection and the penalty paid by the producer to the customer for non-conforming units:

$$
C_{Q}^{1}=q_{0} \times(1-S R) \times c_{n c}+S R \times c_{i}
$$

As in the previous case, the total cost is also a discrete random variable varying within a finite range. Its expected value is derived in the following property.

Property 2. The expression of the expected value of the total cost of policy $\pi_{1}$ is:

$$
E \llbracket T C^{1}\left(X_{0}^{*}, L, S R\right) \rrbracket=E \llbracket T C^{0}\left(X_{0}^{*}, L\right) \rrbracket-\left(c_{h}+c_{t}\right) \times \sum_{s=T-X_{0}^{*}-I}^{T-X_{0}^{*}-1} F_{1}(s)-S R \times\left(c_{n c} \times q_{0}-c_{i}-c_{t} \times t_{i}\right)
$$

Proof. $E \llbracket T C^{0}\left(X_{0}^{*}, L\right) \rrbracket$ can be easily calculated using equation (5) after calculating $X_{0}^{*}$ using equation (9). From equations (11) and (12), it can be easily deduced that:

$$
E \llbracket \Delta_{h}-\Delta_{t} \rrbracket=\left(c_{t}+c_{h}\right) \times E \llbracket \max \left(\min \left(I ; T-X_{0}^{*}-L\right) ; 0\right) \rrbracket-c_{t} \times I
$$

Knowing that $\max \left(\min \left(I ; T-X_{0}^{*}-L\right) ; 0\right)$ is a positive and finite discrete random variable and using equation (6), we get:

$$
\left.E \llbracket \max \left(\min \left(I ; T-X_{0}^{*}-L\right) ; 0\right)\right) \rrbracket=\sum_{s \geq 0}\left(1-\operatorname{Pr} \llbracket \max \left(\min \left(I ; T-X_{0}^{*}-L\right) ; 0\right) \leq s \rrbracket\right)
$$

Knowing that $s \geq 0$, and $I$ and $T-X_{0}^{*}-L$ are independent, so:

$$
\left.\operatorname{Pr} \llbracket \max \left(\min \left(I ; T-X_{0}^{*}-L\right) ; 0\right) \leq s \rrbracket\right)=\operatorname{Pr} \llbracket I>s \rrbracket \times \operatorname{Pr} \llbracket T-X_{0}^{*}-L>s \rrbracket
$$


then:

$$
\begin{aligned}
\left.E \llbracket \max \left(\min \left(I ; T-X_{0}^{*}-L\right) ; 0\right)\right) \rrbracket & =\sum_{s=0}^{I-1} \operatorname{Pr} \llbracket L \leq T-X_{0}^{*}-s-1 \rrbracket \\
& =\sum_{s=0}^{I-1} F_{1}\left(T-s-1-X_{0}^{*}\right) \\
& =\sum_{s=X_{0}^{*}-T+1}^{I+X_{0}^{*}-T} F_{1}(-s) \\
& =\sum_{s=T-X_{0}^{*}-I}^{T-X_{0}^{*}-1} F_{1}(s)
\end{aligned}
$$

Then, the targeted expression of $E \llbracket T C^{1}\left(X_{0}^{*}, L, S R\right) \rrbracket$ is immediate.

\subsection{Policy $2\left(\pi_{2}\right)$ : Integrated production planning and quality control}

In this case, we suppose the same process as in policy $\pi_{1}$ except that the duration of quality control of the finished product is taking into account when optimizing the order release date. Similarly to policy $\pi_{1}$, the total cost is composed of production and quality costs as follows:

$$
T C^{2}(X, L, S R)=C_{P}^{2}+C_{Q}^{2}
$$

where:

$$
\begin{aligned}
& C_{P}^{2}=\left[c_{h} \times\left(T-\min \left(T ; T_{F P}+I\right)\right)+c_{t} \times\left(\max \left(T ; T_{F P}+I\right)-T\right)\right] \\
& \left.C_{Q}^{2}=q_{0} \times(1-S R) \times c_{n c}+S R \times c_{i}\right)
\end{aligned}
$$

$T C^{2}(X, L, S R)$ is also discrete random variable having a finite range of possible values. Thus, the mathematical expectation of the total cost can be determined, as given in if the following property.

Property 3. The expression of the expected value of the total cost of Policy 2 is:

$$
\begin{aligned}
E \llbracket T C^{2}(X, L, S R) \rrbracket= & c_{h} \times\left(\sum_{s=V}^{T-X-I-1} F_{1}(s)\right)+c_{t} \times\left(U+X-T-\sum_{s=T-X-I}^{U-1} F_{1}(s)\right) \\
& +q_{0} \times(1-S R) \times c_{n c}+S R \times c_{i}
\end{aligned}
$$


Proof. From expressions (7) and (8), and by replacing $X$ by $X+I$, we can deduce:

$$
E \llbracket\left(T_{F P}+I\right)^{+} \rrbracket=U+X+I-\sum_{s=T-X-I}^{U-1} F_{1}(s)
$$

and in the same way

$$
E \llbracket\left(T_{F P}+I\right)^{-} \rrbracket=T-\sum_{s=V}^{T-X-I-1} F_{1}(s)
$$

The expected total cost expressed in (14) is also a non-linear objective function to be minimized and for which the Newsboy formula is used to solve it in polynomial time. This method is detailed in the next proposition and an illustrative example is given in section 5.1

Proposition 2. The method based on Newsboy formula gives the following optimality condition for the order release date $X_{2}^{*}$ of Policy 2:

$$
F_{1}\left(T-X_{2}^{*}-I-1\right) \leq \frac{c_{t}}{c_{t}+c_{h}} \leq F_{1}\left(T-X_{2}^{*}-I\right)
$$

where $F_{1}$ is the convolution cumulative distribution function of the total lead time, I is the quality inspection duration, and $c_{t}$ and $c_{h}$ are the unit time tardiness cost and the unit time holding cost, respectively.

Proof. Same as the proof of Proposition 1

\section{Numerical results and discussions}

The proposed three policies described in Section 3 have been coded in $\mathrm{C}++$. The experiments have been carried out on a computer with $2.32 \mathrm{GHz}$ Intel core i7 and 8 GB of RAM memory.

\subsection{Numerical example}

This example is given to illustrate the approach with $m=6$, i.e. 6 production steps. The proportion of defectives generated by the whole process is $q_{0}=0.05$. The cost parameters are as follows: $c_{h}=1, c_{t}=10, c_{i}=0.1$ and $c_{n c}=10$, and the sampling rate $S R$ varies between 0 and 1 . The due date is known and fixed at $T=34$, and the distributions of process steps' lead times are given in Figure 5. 
For all policies, the convolution cumulative distribution function is the same and is equal to: $F_{1}(1)=\ldots=F_{1}(12)=0 ; F_{1}(13)=0.000108 ; F_{1}(14)=0.001836 ; F_{1}(15)=0.011088 ; F_{1}(16)=$ $0.0383976 ; F_{1}(17)=0.0947814 ; F_{1}(18)=0.187245 ; F_{1}(19)=0.314841 ; F_{1}(20)=0.46686 ; F_{1}(21)=$ $0.622675 ; F_{1}(22)=0.760313 ; F_{1}(23)=0.865462 ; F_{1}(24)=0.935485 ; F_{1}(25)=0,974091 ; F_{1}(26)=$ $0.990933 ; F_{1}(27)=0.996905 ; F_{1}(28)=0.999185 ; F_{1}(29)=0.999837 ; F_{1}(30)=0.99996 ; F_{1}(31)=$ $0.999994 ; F_{1}(32)=1$.

Using Propositions (1) and (2), optimal solutions for the three policies are found as follows:

- For policy $\pi_{0}$, the optimal release date $X_{0}^{*}$ is given by the proposition (1) and depends on $c_{h}$ and $c_{t}$. The optimality condition is: $F_{1}(23)=F_{1}\left(34-X_{0}^{*}-1\right) \leq 10 /(1+10) \leq F_{1}\left(34-X_{0}^{*}\right)=$ $F_{1}(24)$. Thus, $X_{0}^{*}=10$.

- For policy $\pi_{1}, X_{1}^{*}=X_{0}^{*}=10$.

- For policy $\pi_{2}$, the optimal release date $X_{2}^{*}$ is given by the proposition (2) and does not depend only on $c_{h}$ and $c_{t}$ but also on $S R$. For example, for $S R=0.4$, the optimality condition is: $F_{1}(23)=F_{1}\left(34-X_{2}^{*}-4-1\right) \leq 10 /(1+10) \leq F_{1}\left(34-X_{2}^{*}-4\right)=F_{1}(24)$. Thus, $X_{2}^{*}=6$.

The results of this example for different values $S R$ are summarized in Table 2 . We note that only the expected cost of Policy $1\left(\pi_{1}\right)$ and Policy $2\left(\pi_{2}\right)$ vary with $S R$. As can be seen from this table, $\pi_{2}$ dominates the others because it is at least as efficient as each them (when $S R=0)$. It is also important to note that $\pi_{1}$ and $\pi_{2}$ allow the reduction of quality cost when sampling rate increases which is the result of reducing non-conformity costs. However, the total expected cost of $\pi_{1}$ is always greater than $\pi_{0}$ 's one because they have the same optimised order release date, and therefore an extra tardiness cost is generated by inspection only in policy $\pi_{1}$.

Table 2.: Results of the numerical example

\begin{tabular}{ccccccccc}
\hline$S R$ & $X_{0}^{*}=X_{1}^{*}$ & $E T C^{0 *}$ & $C Q^{0}$ & $E T C^{1 *}$ & $C Q^{1}$ & $X_{2}^{*}$ & $E T C^{2 *}$ & $C Q^{2}$ \\
\hline 0 & 10 & 4.89969 & 0.5 & 4.89969 & 0.5 & 10 & 4.89969 & 0.5 \\
\hline 0.2 & 10 & 4.89969 & 0.5 & 6.93616 & 0.42 & 8 & 4.81969 & 0.42 \\
\hline 0.4 & 10 & 4.89969 & 0.5 & 14.8713 & 0.34 & 6 & 4.73969 & 0.34 \\
\hline 0.6 & 10 & 4.89969 & 0.5 & 29.2683 & 0.26 & 4 & 4.65969 & 0.26 \\
\hline 0.8 & 10 & 4.89969 & 0.5 & 47.7234 & 0.18 & 2 & 4.57969 & 0.18 \\
\hline 1 & 10 & 4.89969 & 0.5 & 67.5012 & 0.1 & 0 & 4.49969 & 0.1 \\
\hline
\end{tabular}




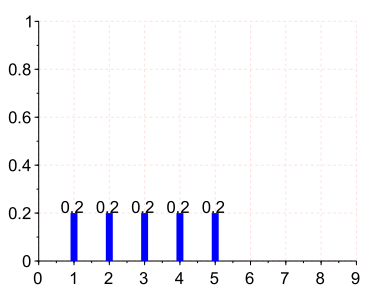

(a) $L_{1}$

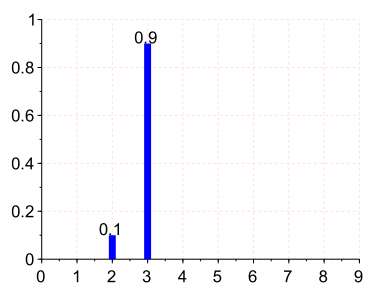

(d) $L_{4}$

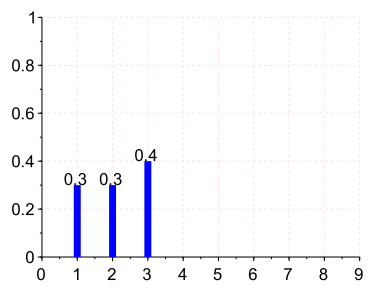

(b) $L_{2}$

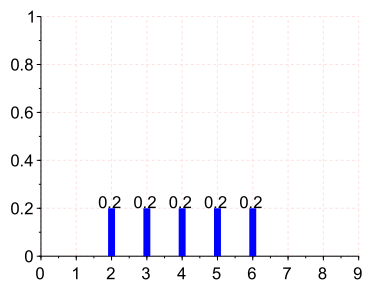

(e) $L_{5}$

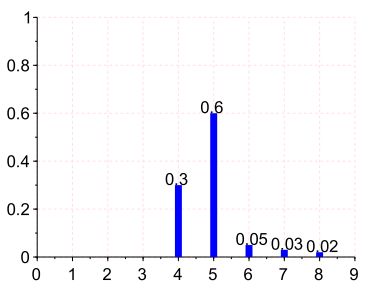

(c) $L_{3}$

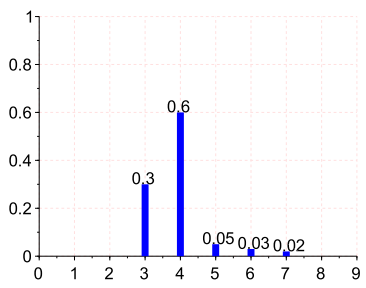

(f) $L_{6}$

Figure 3.: Probability distributions of the lead times $L_{1}$ to $L_{6}$ of the numerical example

\subsection{Comparison of the three policies}

In this subsection, we compare the three quality control policies. We notice firstly that the difference between the expected total costs $E \llbracket T C^{2}\left(X_{2}^{*}, L, S R\right) \rrbracket$ and $E \llbracket T C^{0}\left(X_{0}^{*}, L\right) \rrbracket$ is always equal to $S R\left(c_{i}-\right.$ $\left.q_{0} c_{n c}\right)$. From this, we can deduce analytically that $\pi_{2}$ outperforms $\pi_{0}$ when $c_{i}<q_{0} c_{n c}$ and viceversa. In other words, when the inspection cost is greater than the non-conformity cost of the proportion of defectives, $\pi_{0}$ is dominant, i.e. it is not profitable to make any quality control. Figure 4 illustrates the evolution of the expected total cost of the three policies as function of the number of process steps $m$ for three combinations of inspection and non conformity cost rates: (a) $\rho_{i}=c_{i} / c_{h}=0 \& \rho_{n c}=c_{n c} / c_{h}=20$, (b) $\rho_{i}=10 \& \rho_{n c}=20$ and (c) $\rho_{i}=10 \& \rho_{n c}=1000$. The other parameters are: $q_{0}=0.05, t_{i}=10, S R=0.4$ and $\rho_{t}=c_{t} / c_{h}=100$. Sub-figures (a) and (c) Sub-figure (b) illustrates the case when the policy $\pi_{0}$ dominates the other two policies. We can remark that when the number of process steps increases the gaps between the three policies tend to be constant and that the gap between $\pi_{1}$ and $\pi_{2}$ tends to zero.

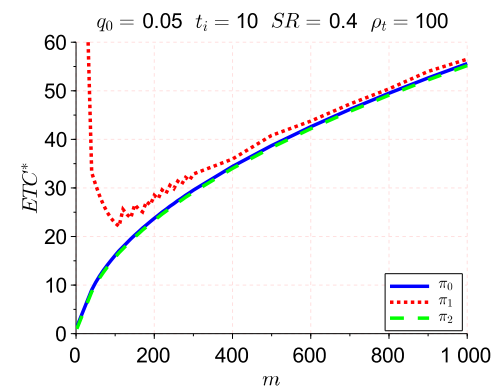

(a) $\rho_{i}=0 \& \rho_{n c}=20$

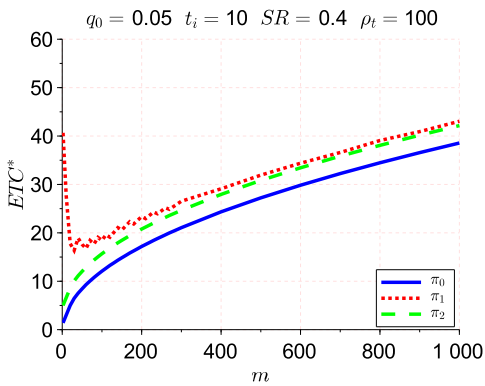

(b) $\rho_{i}=10 \& \rho_{n c}=20$

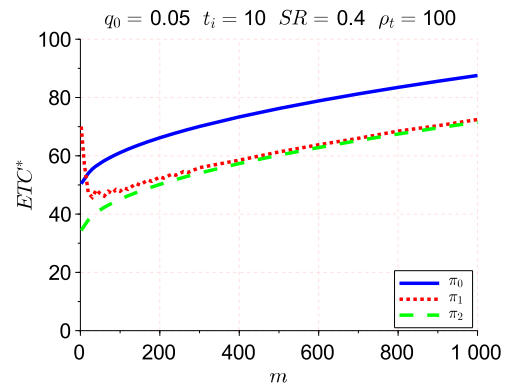

(c) $\rho_{i}=10 \& \rho_{n c}=1000$

Figure 4.: Comparison of the three policies: ETC as function of $m$ 
In order to go farther in the analyse of the relative performance of the three policies, we compare them two-by-two according to their expected total costs. To do so, we use the following performance criteria:

- The gap between policy $\pi_{0}$ and policy $\pi_{1}$ :

$$
G A P 01(\%)=100 \times \frac{E T C^{0}-E T C^{1}}{E T C^{1}}
$$

- The gap between policy $\pi_{0}$ and policy $\pi_{2}$ :

$$
G A P 02(\%)=100 \times \frac{E T C^{0}-E T C^{2}}{E T C^{2}}
$$

- The gap between policy $\pi_{1}$ and policy $\pi_{2}$ :

$$
G A P 12(\%)=100 \times \frac{E T C^{1}-E T C^{2}}{E T C^{2}}
$$

Figure 5, Figure 6 and Figure 7 show the variation of the gaps between the three policies as function of the sampling rate and non conformity cost rate $\left(\rho_{n c}=c_{n c} / c_{h}\right)$ for different values of inspection cost rate $\left(\rho_{i}=c_{i} / c_{h}\right)$. Firstly, it can be seen the trivial fact that, if non-conformity penalty is negligible $\left(\rho_{n c} \leq 1\right)$, policy $\pi_{0}$ outperforms both other policies $\pi_{1}$ and $\pi_{2}$, whatever are the inspection cost and the sampling rate.

Figure 5 illustrates that GAP01 varies between $-100 \%$ and $20 \%$. When $\rho_{i}$ is less than or equal to 30 , there exists a non-conformity cost for which the gap is maximum only for specific value of sampling rate. This fact can be used to help decision maker regarding the inspection capacity needed.

From Figure 6, it can bee seen that, for $\rho_{i} \leq 30$, increasing the sampling rate improves the performance of $\pi_{2}$ compared to $\pi_{0}$. In contrary, when inspection cost increases, assuming nonconformity cost becomes better than doing inspection.

Figure 7 shows the evolution of the gap between policies $\pi_{1}$ and $\pi_{2}$ as function of the sampling rate for different values of the non-conformity and inspection costs. It can been seen that $\pi_{2}$ always outperforms $\pi_{1}$ whatever are the cost parameters and the sampling rate. In fact, GAP12(\%) is always positive and increases with the sampling rate. We remark also that this gap is globally less important when the inspection cost increases: it becomes less than $7 \%$ when $\rho_{i}=1000$, whatever are the sampling rate and the non-conformity cost. It outlines the importance of integrating quality control in supply and production planning. 


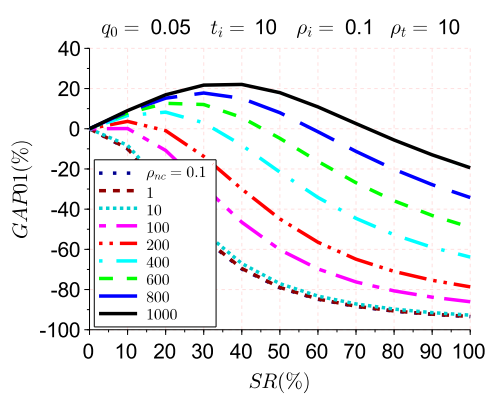

(a) $\rho_{i}=0.1$

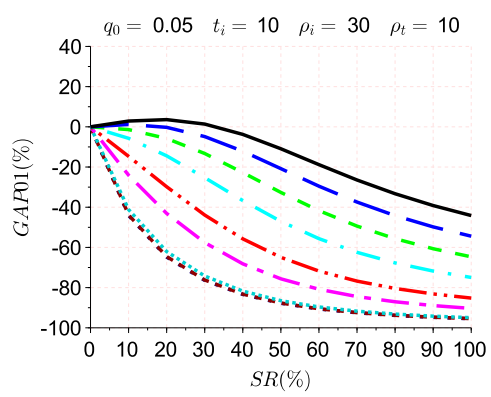

(d) $\rho_{i}=30$

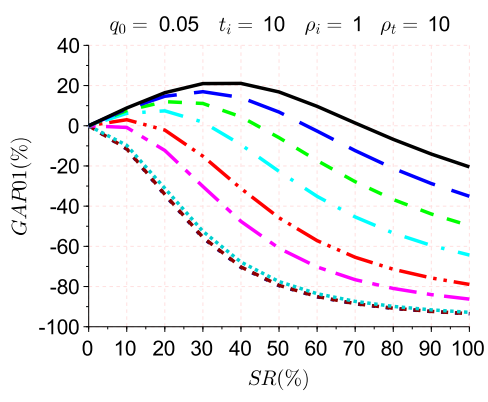

(b) $\rho_{i}=1$

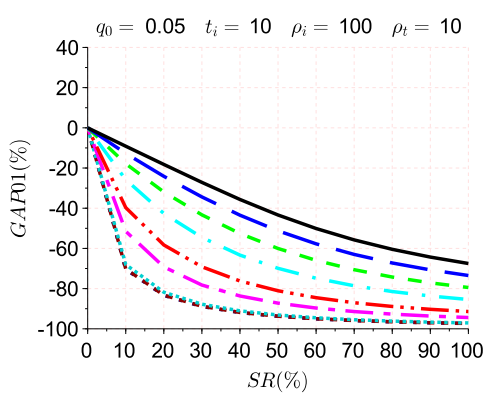

(e) $\rho_{i}=100$

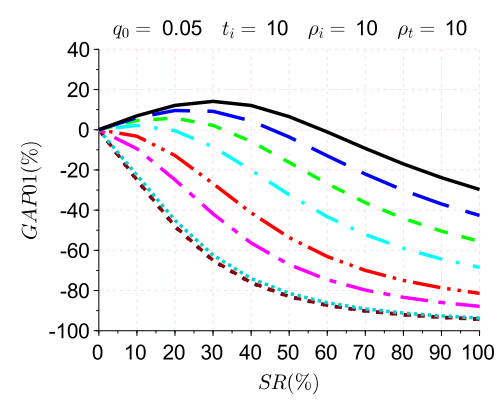

(c) $\rho_{i}=10$

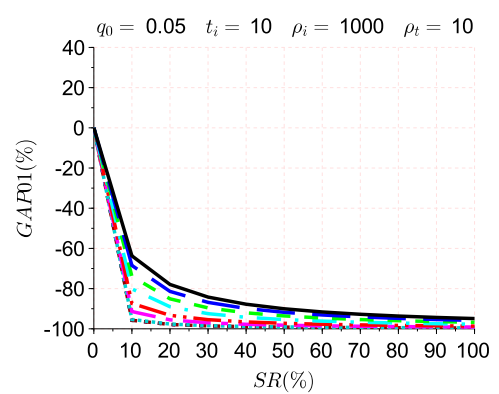

(f) $\rho_{i}=1000$

Figure 5.: Performance of policy $\pi_{0}$ compared to policy $\pi_{1}$

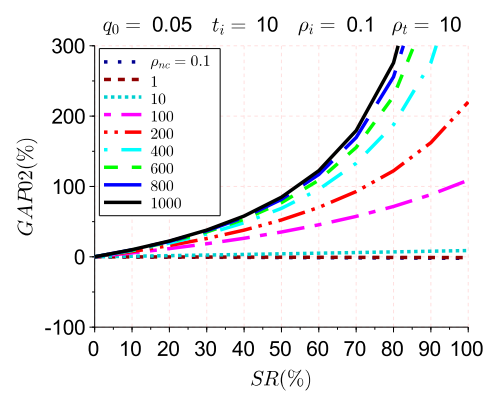

(a) $\rho_{i}=0.1$

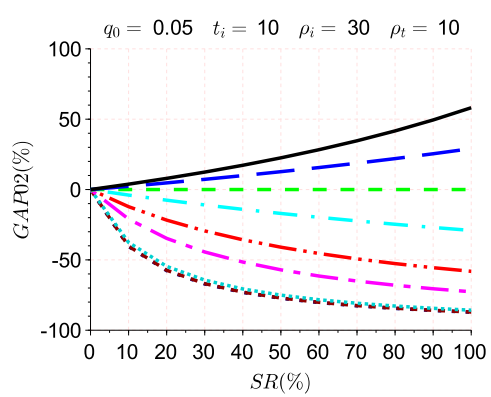

(d) $\rho_{i}=30$

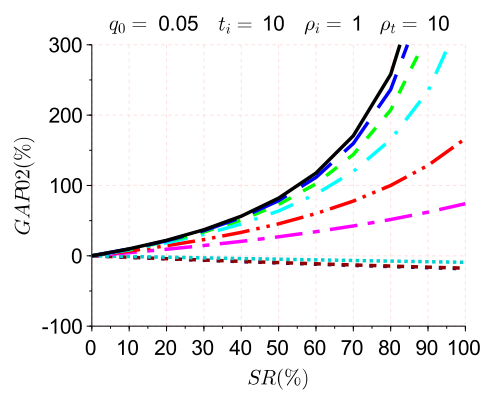

(b) $\rho_{i}=1$

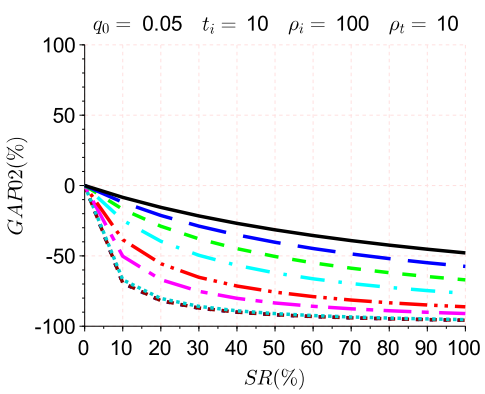

(e) $\rho_{i}=100$

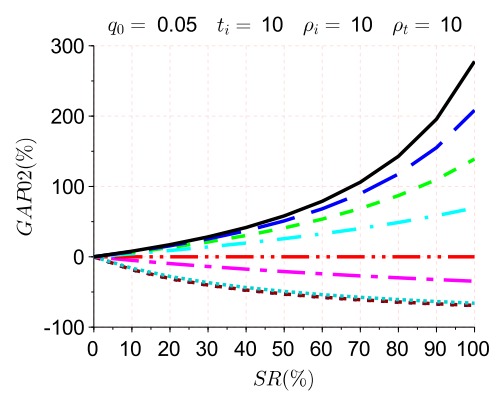

(c) $\rho_{i}=10$

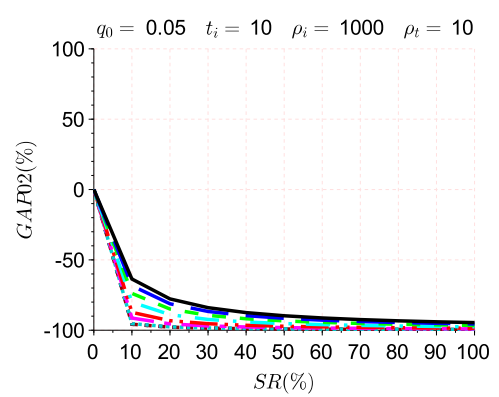

(f) $\rho_{i}=1000$

Figure 6.: Performance of policy $\pi_{0}$ compared to policy $\pi_{2}$

It can be concluded that even if it is clear that quality control is not always economically beneficial when the cost of quality is much higher than the non-conformity penalties, companies may be sometimes obliged to set up quality control (warranties, others usages of the information brought by inspection, certification, etc). In this case it is proven that integrating the quality control into the production planning is advisable to minimize the cost of production. 


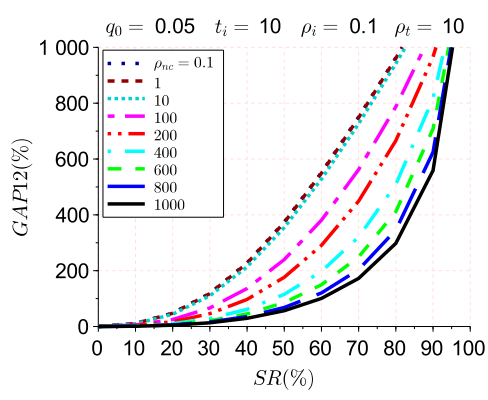

(a) $\rho_{i}=0.1$

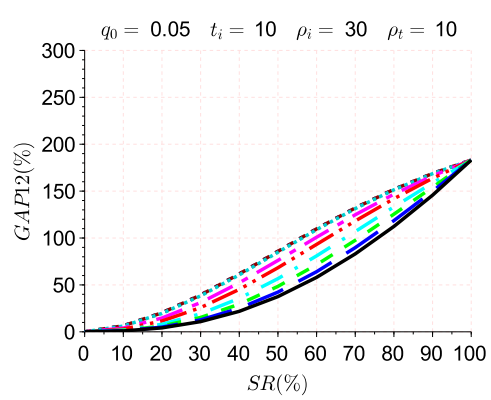

(d) $\rho_{i}=30$

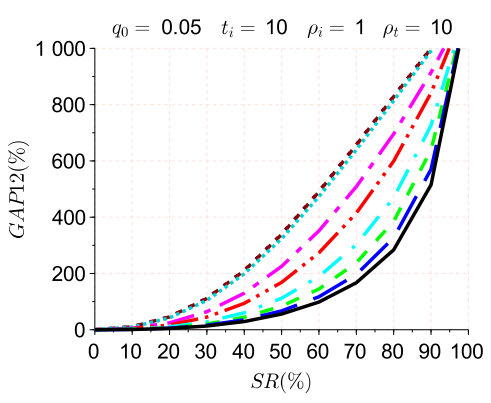

(b) $\rho_{i}=1$

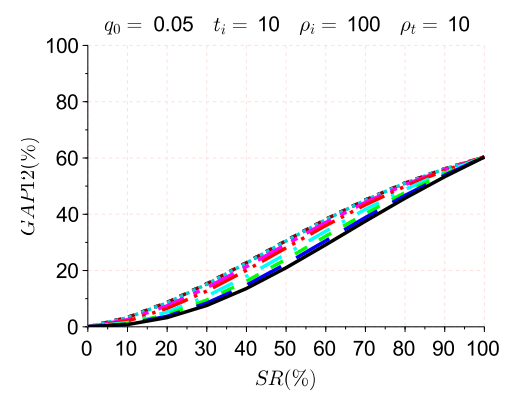

(e) $\rho_{i}=100$

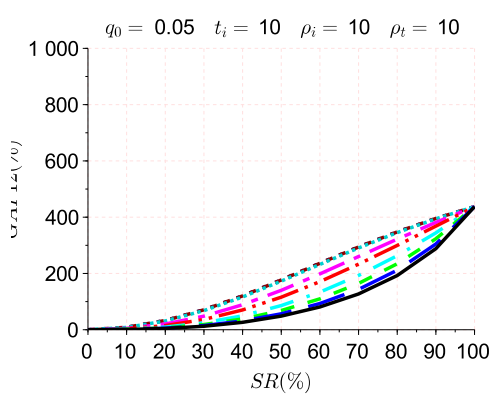

(c) $\rho_{i}=10$

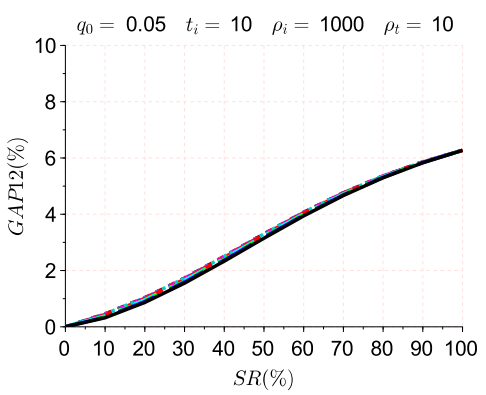

(f) $\rho_{i}=1000$

Figure 7.: Performance of policy $\pi_{1}$ compared to policy $\pi_{2}$

The previous results showed that, when the final inspection is mandatory to improve the quality level of the final product delivered to the final customer, the final inspection should be considered as a final process step when optimising the planned lead time. This is proved by the fact that policy $\pi_{2}$ always dominates policy $\pi_{1}$. Moreover, policy $\pi_{2}$ and policy $\pi_{1}$ cover policy $\pi_{0}$, because the latter is a special case of the first ones when $S R=0$. That is why, we focus only on policy $\pi_{2}$ in the following experiments to analyse the effects of two parameters on the effectiveness and the robustness of the proposed approach.

\subsection{Impact of the number of process steps on policy 2}

To analyse the performance of $\pi_{2}$, we perform several experiments and variate the number of process steps. For more than 30 process, the probability distribution of the delivery date of the finished product tends to a Normal distribution. This well known phenomena, which is explained theoretically by the central limit theorem, is illustrated in in Figure 8 where the number of process steps $(m)$ varies between 10 and 100 . The lead time of each process varies between 3 and 7 , and its probability distribution is illustrated in the same figure. Computationally, our approach remains efficient even if the total number of process steps is large. This is shown in Figure 9 which gives the evolution of CPU times as function of $m$. It can be seen that the CPU time remains less than 14 seconds for a serial production process with 6000 steps, even if it is unlikely to have such a number of process steps in practice. 
We note that, even if our method is an effective way to optimize the ETC of the production process under uncertainty of lead time and quality control, the effect of lead time uncertainty increases as function of the number of steps. In fact, even if the variances of steps' lead times are low, the more is number of process steps, the more is the variability of the delivery date. Therefore, the occurrence of extreme values of the latter can lead to extremely high costs. The solution to this problem is to cope with the sources of these uncertainties by trying to eliminate them as much as possible.

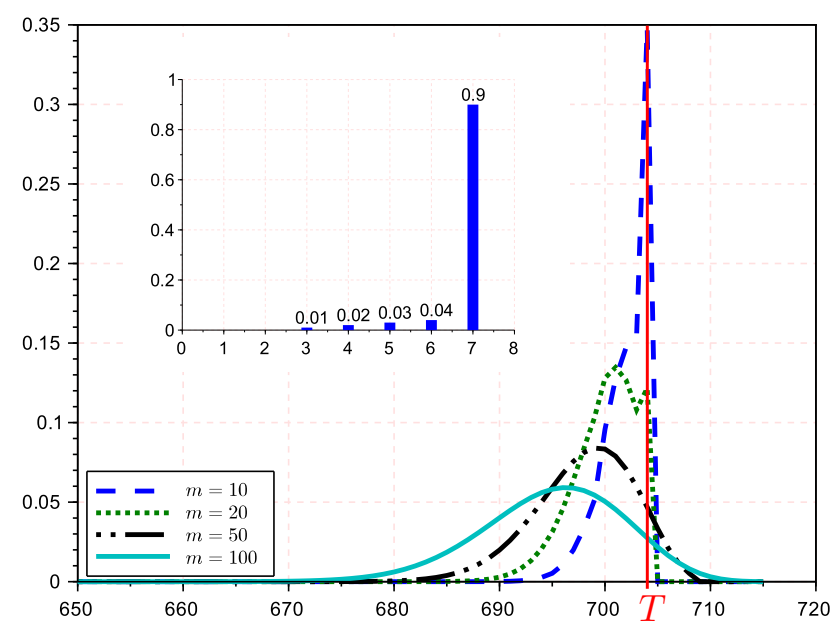

Figure 8.: Effect of $m$ on the probability distribution of the delivery date of policy $\pi_{2}\left(X_{2}^{*}+L+I\right)$

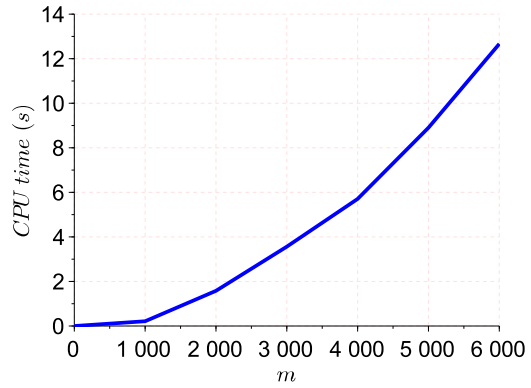

Figure 9.: Effect of $m$ on the CPU Time of policy $\pi_{2}$

\section{$5.4 \quad$ Robustness of policy 2}

In order to analyse the effect of lead times variability on the robustness of Policy 2, we carried out several experiments. We consider a system with $m=6$ process steps having the same probability distribution of their lead times (see Figure 10-(a)) and the following parameters: $c_{h}=1, \rho_{t} \in$ $\{0.1,1,2,5,10,30,50,100\}, \rho_{i}=0, \rho_{n c}=20$ and $S R=0.4$. Then, we proceed by varying the 
variance of process steps' lead times $(\triangle V A R)$ between $-75 \%$ and $75 \%$ (Figure 10-(b) to $-(\mathrm{g})$ ) compared to the reference case (Figure 10-(a)) .

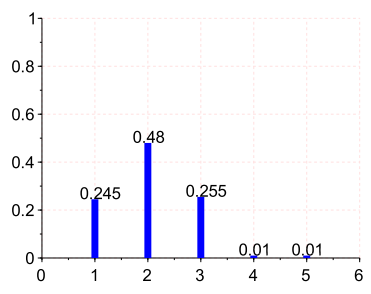

(a) $f_{L_{j}}$

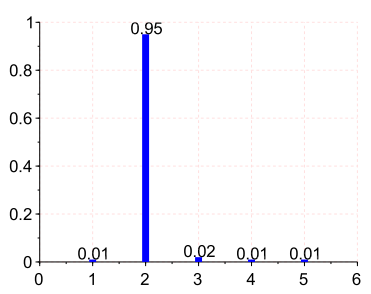

(b) $f_{L_{j}}^{\Delta V A R=-75 \%}$

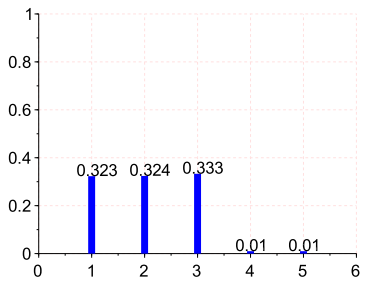

(e) $f_{L_{j}}^{\Delta V A R=+25 \%}$

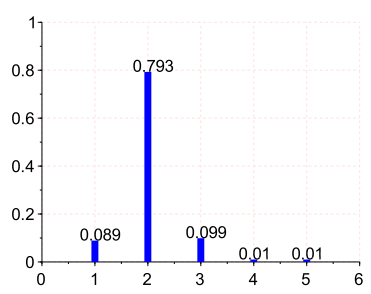

(c) $f_{L_{j}}^{\Delta V A R=-50 \%}$

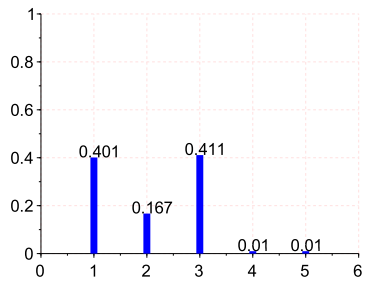

(f) $f_{L_{j}}^{\Delta V A R=+50 \%}$

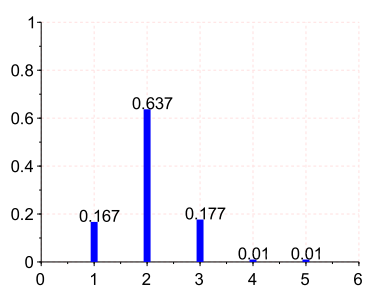

(d) $f_{L_{j}}^{\Delta V A R=-25 \%}$

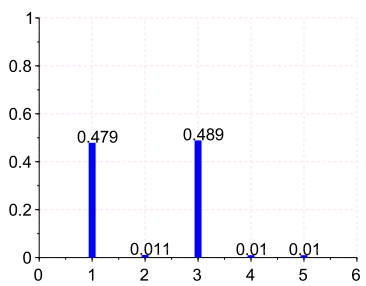

(g) $f_{L_{j}}^{\Delta V A R=+75 \%}$

Figure 10.: Probability distributions of the lead times $L_{j}$ and the modified distributions corresponding to different levels of $\triangle V A R$

Figure 11 represents the probability distributions of the delivery date for different levels of variance variation $(\triangle V A R)$ for $m=6$, while using the same order release date $X_{2}^{*}$ optimised for the reference case. From the zooming in at the right of the delivery date $T$, it can be seen that a positive variation of $\triangle V A R$ leads to an increased probability of backlogging, and a decreased probability of positive inventory level, and vice-versa.

Figure 12 shows the effect of the variation of the variance of lead times on the ETC as function of the backlogging cost rate $\rho_{t}$, for a serial production system with $m=100$ process steps. We firstly notice that, for a relatively low variation of the variance of lead times $(\triangle V A R=-25 \%$ and $25 \%)$, the variation of ETC increases slightly with the backlogging cost rate $\rho_{t}$ and remains at around $\pm 10 \%$. We also notice that, for an increased $\rho_{t}$, the variation of ETC is clearly more significant, when $\triangle V A R$ is positive than when it is negative. However, when the backlogging cost is relatively low $\left(0<\rho_{t} \leq 10\right)$, a variance reduction $(\Delta V A R<0)$ has more significant impact on the ETC. It means that, it should be better to overestimate the variability of the lead times. It is worthwhile to 
mention that it is highly important to obtain reliable statistical data to get a good estimate of the probability distributions of lead times and to track and eliminate as much as possible the sources of the uncertainty.

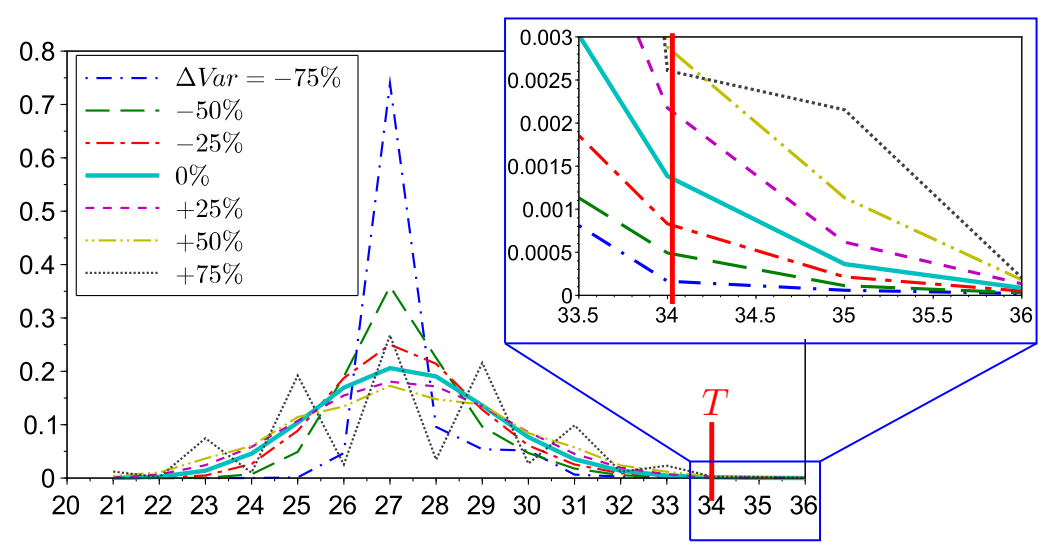

Figure 11.: Probability distribution of the delivery date for different levels of variance variation

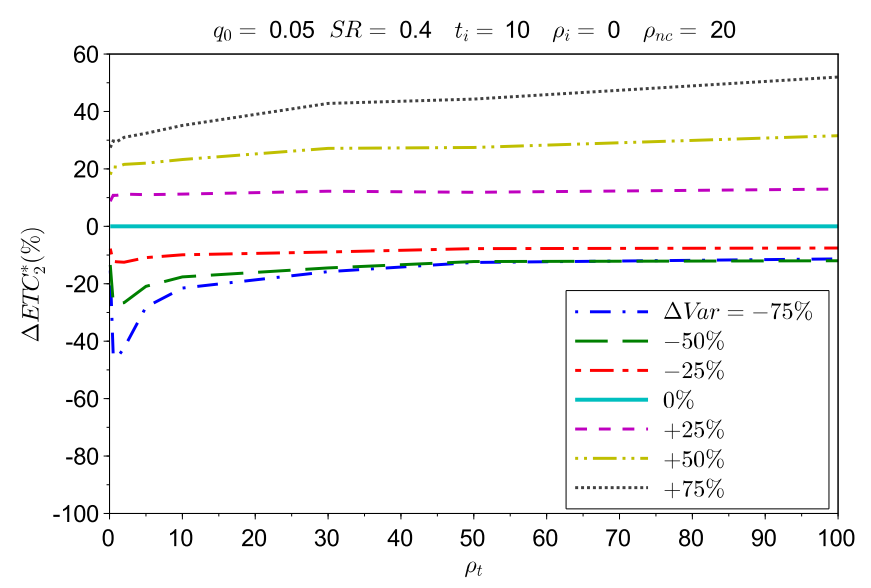

Figure 12.: Effect of lead time variance on the expected total cost of policy $\pi_{2}$

\section{Conclusion and Perspectives}

In this work, the main purpose was to integrate quality control to supply and production planning under uncertainties of lead times and finished product quality in linear systems. We consider a single-period production planning model with a known demand and a known due date. To our best of knowledge, this is the first study to investigate serial systems with quality control and uncertainty of lead times. We propose an analytical model to analyse three quality control policies: i) without quality control, ii) with quality control but inspection duration is not integrated when optimising the release date, and iii) with quality control and the inspection duration is included when optimising the release date. The proposed model optimises, for a given quality control policy, 
the order release date while minimizing the expected total cost which is the sum of inventory holding, backlogging and quality costs.

In order to test the advantages of each quality control policy, several experiments were carried out. As shown from results analysis, there is a significant economical advantage of integrating quality control planning at the early stage of supply and production planning decisions. The robustness of the solution provided by this model is also analysed regarding the variance of lead times. It can been noticed that the ETC is more affected when the variability increases than when it decreases. This implies that, if the variability of lead times cannot be reduced and/or precisely estimated, it is preferable to overestimate it in order to avoid the situation with a high increase of the actual total cost compared to what was expected.

Moreover, the proposed model may be helpful during contract negotiation step with a customer in an ETO environment with successive processing steps. When a given client orders a tailored finished product, a decision maker can estimate the total lead time and negotiate (i) the planned due date for client delivery, (ii) the unit non-conformity cost and (iii) the unit backlogging cost. According to the negotiated parameters and using our approach, he can decide the date when the first process starts and which quality control policy to adopt. Furthermore, the proposed approach can profit from the abundance of real-time data in the context of Industry 4.0 environment which will allow to improve the precision of uncertainty estimation and to make it possible to adopt more reactive, coordinated and integrated decision making approaches to optimizing all kinds of operational activities in the SC.

Several perspectives to this work are identified and are to be explored. Firstly, it will be interesting to investigate the case where it is possible to make and to coordinate quality control decisions at each process step and where the quality of the finished product depends on the sampling decisions of all process steps. The second perspective to this work is to extend this model by integrating other activities such as maintenance and by considering other kinds of production systems such as assembly systems, where the finished product is assembled from several components and semifinished products. Finally, the objective is to generalize the model for the case of multi-period planning, multi-product, and multi-stage assembly systems, and to propose efficient optimization techniques for them.

\section{References}

Aloulou, M.A., A. Dolgui, and M.Y. Kovalyov. 2014. "A bibliography of non-deterministic lot-sizing models." International Journal of Production Research 52 (8): 2293-2310. 
Ben-Ammar, O., Dolgui A., and D.D. Wu. 2018. "Planned lead times optimization for multi-level assembly systems under uncertainties." Omega 78: $39-56$.

Ben-Ammar, O., B. Bettayeb, and A. Dolgui. 2017. "Supply planning optimization for linear production system with stochastic lead-times and quality control." In International Conference on Industrial Engineering and Systems Management (IESM 2017), .

Ben-Ammar, O., H. Marian, A. Dolgui, and D.D. Wu. 2014. "Reducing the research space of possible order release dates for multi-level assembly systems under stochastic lead times." In IFIP International Conference on Advances in Production Management Systems, 368-374. Springer.

Bettayeb, B., S.J. Bassetto, and M. Sahnoun. 2014. "Quality control planning to prevent excessive scrap production." Journal of Manufacturing Systems 33 (3): 400-411.

Bettayeb, B., N. Brahimi, and D. Lemoine. 2017. "Integrated dynamic single item lot-sizing and quality inspection planning." International Journal of Production Research 1-17.

Buzacott, J.A., and JG. Shanthikumar. 1994. "Safety stock versus safety time in MRP controlled production systems." Management science 40 (12): 1678-1689.

Colledani, M., and Tullio T. 2006. "Impact of Quality Control on Production System Performance." CIRP Annals - Manufacturing Technology 55 (1): 453-456.

Díaz-Madroñero, M., J. Mula, and D. Peidro. 2014. "A review of discrete-time optimization models for tactical production planning." International Journal of Production Research 52 (17): 5171-5205.

Ding, H., L. Benyoucef, and X. Xie. 2005. "A simulation optimization methodology for supplier selection problem." International Journal of Computer Integrated Manufacturing 18 (2-3): 210-224.

Dolgui, A., O. Ben-Ammar, F. Hnaien, and M.A. Louly. 2013. "A state of the art on supply planning and inventory control under lead time uncertainty." Studies in Informatics and Control 22 (3): 255-268.

Dolgui, A., G. Levin, and M.A. Louly. 2005. "Decomposition approach for a problem of lot-sizing and sequencing under uncertainties." International Journal of Computer Integrated Manufacturing 18 (5): $376-385$.

Dolgui, A., and C. Prodhon. 2007. "Supply planning under uncertainties in MRP environments: A state of the art." Annual Reviews in Control 31 (2): 269-279.

Elhafsi, M. 2002. "Optimal leadtimes planning in serial production systems with earliness and tardiness costs." IIE transactions 34 (3): 233-243.

Gong, L., T. de Kok, and J. Ding. 1994. "Optimal leadtimes planning in a serial production system." Management Science 40 (5): 629-632.

Guide, V.R., and R. Srivastava. 2000. "A review of techniques for buffering against uncertainty with MRP systems." Production Planning \& Control 11 (3): 223-233.

Jansen, S., Z. Atan, I. Adan, and T. de Kok. 2018. "Setting optimal planned leadtimes in configure-to-order assembly systems." European Journal of Operational Research .

Kim, J.G., D. Chatfield, T.P. Harrison, and J.C. Hayya. 2006. "Quantifying the bullwhip effect in a supply chain with stochastic lead time." European Journal of operational research 173 (2): 617-636. 
Kim, J.G., D. Sun, X.J. He, and J.C. Hayya. 2004. "The (s, Q) inventory model with Erlang lead time and deterministic demand." Naval Research Logistics (NRL) 51 (6): 906-923.

Kuang, H., S.J. Hu, and J. Ko. 2016. "A dynamic programming approach to integrated assembly planning and supplier assignment with lead time constraints." International Journal of Production Research 54 (9): 2691-2708.

Matsuura, H., and H. Tsubone. 1993. "Setting planned leadtimes in capacity requirements planning." Journal of the Operational Research Society 44 (8): 809-816.

Matsuura, H., H. Tsubone, and M. Kanezashi. 1996. "Setting planned lead times for multi-operation jobs." European journal of operational research 88 (2): 287-303.

May, B. I., M.P. Atkinson, and G. Ferrer. 2017. "Applying inventory classification to a large inventory management system." Journal of Operations and Supply Chain Management 10 (1): 68-86.

Petrovic, D., R.t Roy, and R. Petrovic. 1998. "Modelling and simulation of a supply chain in an uncertain environment." European journal of operational research 109 (2): 299-309.

Rosenblatt, M.J., and H.L. Lee. 1986. "Economic Production Cycles with Imperfect Production Processes." IIE Transactions 18 (1): 48-55.

Schemeleva, K., X. Delorme, and A. Dolgui. 2018. "Evaluation of solution approaches for a stochastic lot-sizing and sequencing problem." International Journal of Production Economics 199: 179-192.

Simangunsong, E., L.C. Hendry, and M. Stevenson. 2012. "Supply-chain uncertainty: a review and theoretical foundation for future research." International Journal of Production Research 50 (16): 4493-4523.

Tang, C.S. 1990. "The Impact of Uncertainty on a Production Line." Management Science 36 (12): 15181531.

Van der Vorst, J.G.A.J., and A.J.M. Beulens. 2002. "Identifying sources of uncertainty to generate supply chain redesign strategies." International Journal of Physical Distribution \& Logistics Management 32 (6): 409-430.

Weeks, J.K. 1981. "Optimizing planned lead times and delivery dates." In 21st Annual Conference Proceeding, American Production and Inventory Society, 177-188.

Whybark, D.C., and J.G. Williams. 1976. "Material requirements planning under uncertainty." Decision sciences 7 (4): 595-606.

Yano, C.A. 1987a. "Setting planned leadtimes in serial production systems with tardiness costs." Management science 33 (1): 95-106.

Yano, C.A. 1987b. "Planned leadtimes for serial production systems." IIE transactions 19 (3): 300-307. 